\title{
Permanent Magnet Synchronous Motor loss Analysis Based on The Time Stepping Finite Element Method
}

\author{
Chunhong Deng ${ }^{1, a}$, Li Tian ${ }^{2, b}$, Meng Wang ${ }^{1, \mathrm{c}}$ \\ ${ }^{1}$ Department of Information Engineering, Anhui Technological College of Machinery and Electricity, \\ WuHu, China \\ ${ }^{2}$ School of Electrical Engineering, Anhui Polytechnic University, WuHu, China \\ aahjddch@126.com, ${ }^{b}$ tli1116@163.com, cwilltg@sina.com
}

Key words: Halbach iron loss. Compound motor. magnetic gear

Abstract : Based on the study of radial magnetized magnetic gears of permanent magnet synchronous motor, put forward a new type of Halbach magnetic composite gear motor to improve the efficiency of the composite motor driving system. Build finite element analysis model, based on the analysis of the distribution of air gap flux density, comprehensive consideration of the effects of harmonic magnetic field and the chute, time stepping finite element method is applied on the research of Halbach magnetic gear composite motor iron loss distribution. The results show that, compared with the traditional radial type structure, Halbach composite magnetic gear motor with small iron loss, high efficiency. loss of the motor is affected by the magnetic block, the width of slot, which lays a foundation for improving the efficiency of this kind of motor.

\section{Introduction}

Compared with the traditional mechanical gears, the magnetic field modulated magnetic gear is used to change the air gap magnetic field, the inner and outer permanent magnets are involved in the transmission of torque. There is no mechanical friction in the process of transmission, which makes the transmission device with many advantages, such as large output torque density, low noise, no mechanical loss, high transmission efficiency. In view of the advantages, experts and scholars combined the magnetic field modulation magnetic gear with permanent magnet motor, invented magnetic composite gear motor, which has the low-speed and high torque direct drive mode, and also realized the application of magnetic gear of the internal space, which improved the efficiency of the whole power system. In addition, this kind of motor has the advantages of high power density, high power factor, and can be used in electric vehicles, which can save the mechanical gearbox, reduce the volume, improve the efficiency of transmission, and increase the utilization rate of fuel ${ }^{[1][2]}$, Applied to wind power generator, it can replace the traditional mechanical gear, and solve the problem of speed matching between the wind turbine and generator, and improve the efficiency of the wind power system.

Compared with the ordinary permanent magnet motor, there are two permanent magnets in the inner and outer of the motor, and also a large magnetic field in the inner and outer permanent magnets. Accurate calculation and analysis of iron loss is significant to the application of this kind of motor. Literature 3 is the first doctoral thesis of the magnetic gear motor, a new type of magnetic gear motor, using analytical method to study the variation of the magnetic density of each part, and some conclusions are drawn. The magnetic density distribution is analyzed by literature 4 , In the literature, the magnetic field and the electromagnetic torque are calculated by the global analytic method, and the obtained Halbach magnetic gear is more close to the sine wave. Literature 5 shows the influence of the structure parameters on the mechanical and magnetic properties of the permanent magnetic gear and the permanent magnet brushless motor, to verify the effectiveness of the design by using the finite element method. The above literatures show more of that the static characteristics and the theory of the magnetic gear motor,but less research on the iron loss of the composite motor.

In this paper,proposing a new hybrid electric motor with Halbach topology, Iron loss calculation model of composite motor is established, studying on the magnetic density distribution 
and the change of the typical position of the composite motor, calculating the loss distribution of the magnetic field and permanent magnet in the composite motor. With the application of the 2D finite element method, proposed Some methods for reducing the iron loss of the composite motor, which are the basis for improving the efficiency of the magnetic gear motor.

\section{Magnetic gear transmission mechanism}

The model of the magnetic gear motor is shown in Figure 1. Figure (a) is a permanent magnet with a radial structure, Figure (b) is a permanent magnet with a Halbach structure. The composite motor is a double-rotor structure, The innermost is a outer rotor permanent magnet synchronous motor with 3 poles, the number of inner rotor magnetic poles is 4 , and it is 17 for outer rotor poles, and the magnetic field is between the magnetic pole. According to the mechanism of magnetic gear transmission, the number of the intermediate transfer block is the sum of the inner rotating pole. The angular velocity of the inner and outer rotor is as follows.

$$
\omega_{2}=-\frac{p_{1}}{p_{2}} \omega_{1}
$$

Where: $p_{1}$ is outer rotor pole number, $p_{2}$ is inner rotor pole number, $\omega_{1}$ is outer rotor angular velocity, $\omega_{2}$ is inner rotor angular velocity.


Fig. 1 Model of magnetic gear motor. a-Radial structure, $b$-Halbach structure

\section{Air gap magnetic density calculation}

\section{Finite element model}

The electromagnetic field analysis is the basis of the analysis of motor loss, heat, etc., and the premise of that the motor can run reliably.

Based on the study of magnetic gear transmission composite motor principle, establish finite element analysis model of the motor, and no-load magnetic field distribution of the motor is shown in figure 2.
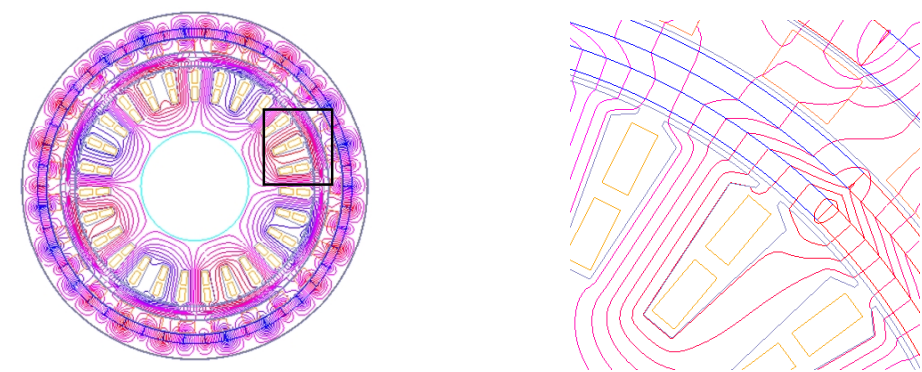

Fig. 2 Magnetic gear composite magnetic flux distribution. 1-Radial structure, 2-Local distribution 

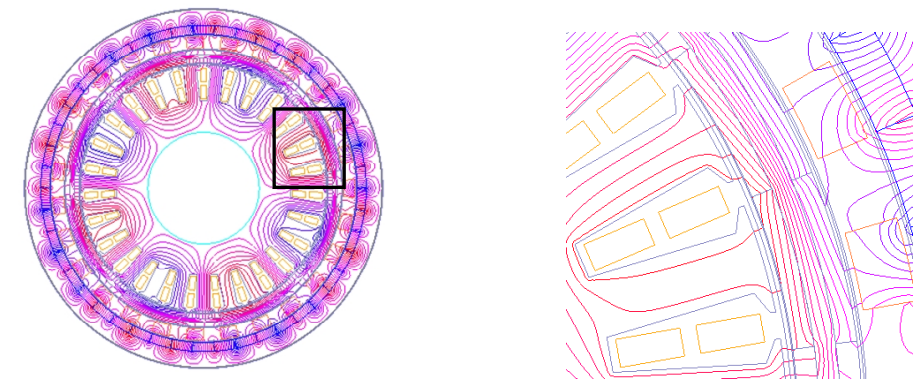

Fig. 3 Magnetic gear composite magnetic flux distribution. 1 -Radial structure, 2 -Local distribution

Figure 2 and 3 show that compared with ordinary radial magnetization, inner and outer layer opermanent magnet with application of Halbach has magnetic composite motor density decrease and air gap flux increase. The permanent magnet synchronous motor and magnetic gear are more powerful, and the dynamic performance of the motor is improved.

\section{Air gap magnetic density analysis}

Halbach combined a magnetic gear motor with permanent magnet layer,which applied Halbach magnetized, the permanent magnets are in the same shape, magnetization with regularity. It is assumed that each pole of the motor is composed of $n$ permanent magnets, First one along the positive direction of magnetization direction as $\mathrm{X}$ axis, other permanent magnets magnetization direction of the magnetization in $\mathrm{X}$ and $\mathrm{Y}$ components, which can be expressed by the following equation.

$$
\begin{aligned}
& M_{x}(i)=M \cos \left((1 \pm p) \frac{360(i-1)}{2 p n}\right) \\
& M_{y}(i)=M \sin \left((1 \pm p) \frac{360(i-1)}{2 p n}\right)
\end{aligned}
$$

Where: $\mathrm{M}$ is magnetization, $\mathrm{P}$ is pole pairs.

Among them, the internal magnetic field is "+", the external magnetic field is "-".

Based on the characteristics of the composite motor, the motor has three layers of air gap, The magnetic flux density of the motor is analyzed and calculated. The three - layer air gap magnetic waveform and harmonic analysis are as follows.
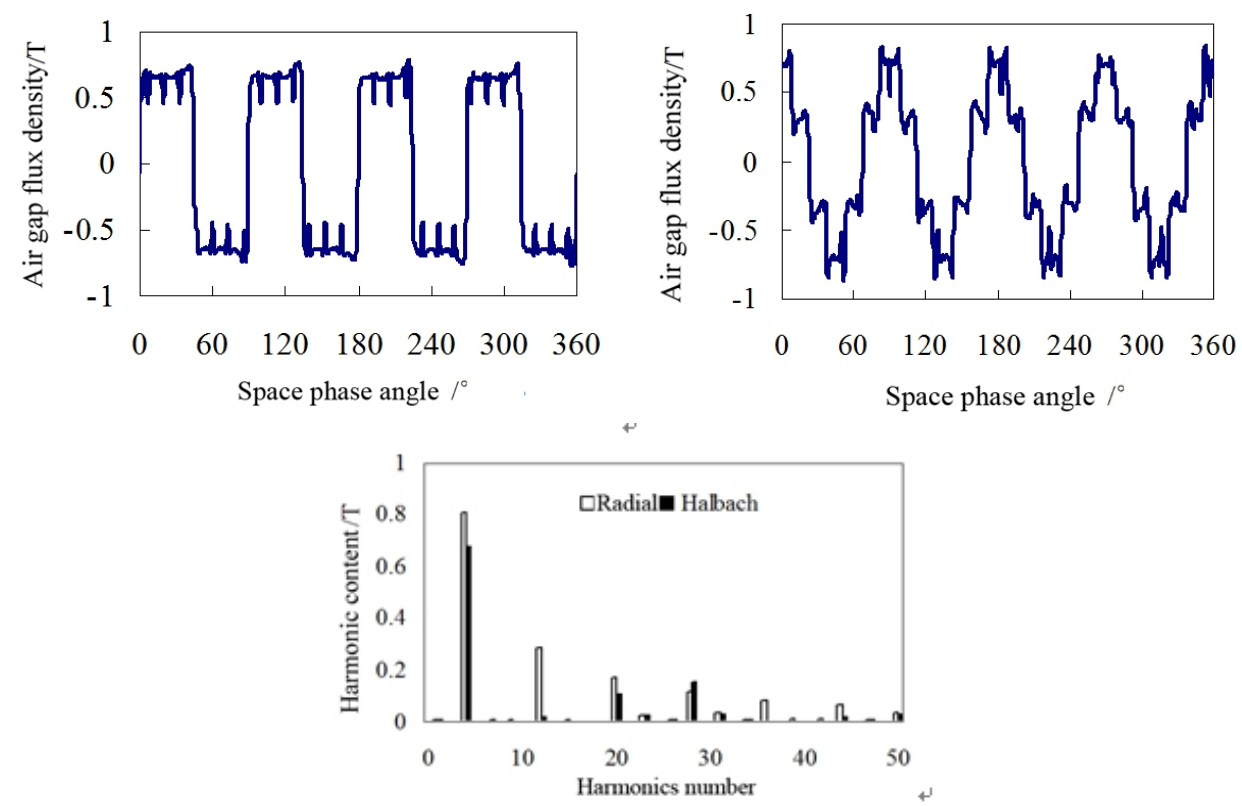

Fig. 4 Motor inner air gap magnetic density. 1 -Radial magnetization, 2-Halbach magnetization, 3 -Harmonic analysis chart 

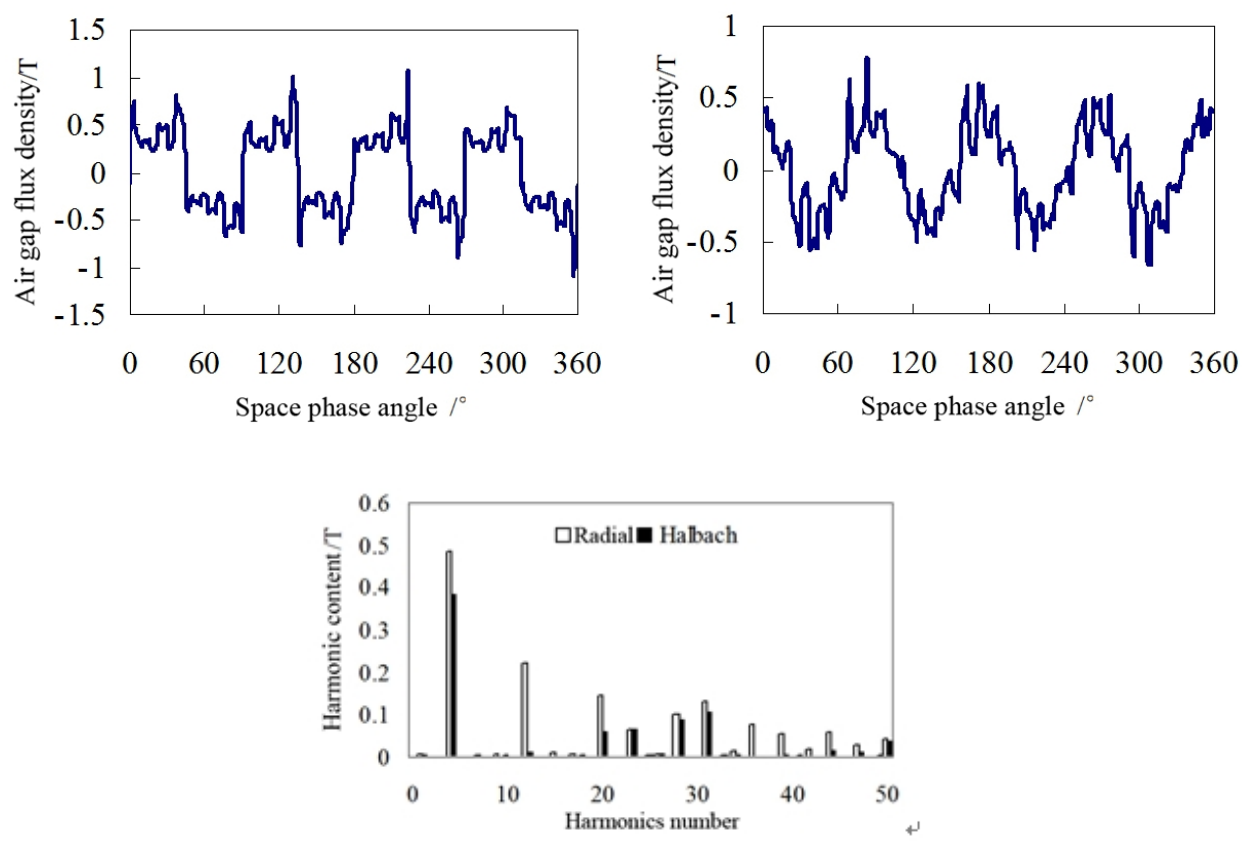

Fig. 5 The middle air gap magnetic field of motor. 1 -Radial magnetization, 2 -Halbach magnetization, 3 -Harmonic analysis chart
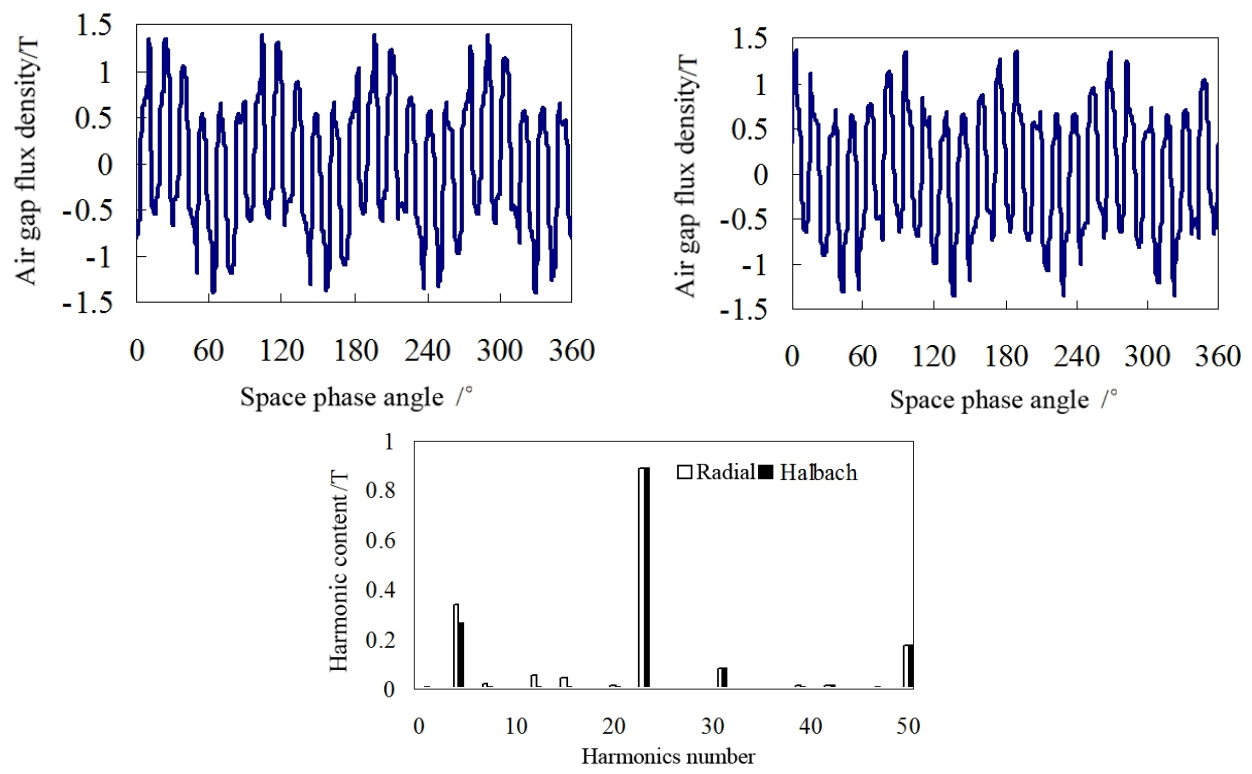

Fig. 6 The outer air gap of the motor. 1-Radial magnetization, 2-Halbach magnetization, 3-Harmonic analysis chart

In Figure 4, 5, 6, by Halbach magnetized, the magnetic field strength of three layers of air gap has been effectively improved, but the amplitude of harmonics is greatly reduced, the sinusoidal air gap flux density is greatly enhanced, the magnetic flux leakage can be controlled effectively. Compared with the fundamental amplitude, although the radial magnetized air gap flux density decreases slightly, the comprehensive performance of the motor is increased.

\section{Iron loss analysis}

In general, the iron loss of the motor is divided into two parts, eddy current loss and hysteresis loss.Using the finite element analysis software, the magnetic density distribution of the composite motor is calculated, and the calculation formula of the iron loss is as follows.

$$
P_{e}=\oint \sum_{n} \rho K_{e}(n f)^{2}\left(B_{n r}^{2}+B_{n t}^{2}\right) d V
$$




$$
P_{h}=\oint \sum_{n} \rho K_{h}(n f)^{2}\left(B_{n r}^{2}+B_{n t}^{2}\right) d V
$$

Where: $\rho$ is the core density, $\mathrm{n}$ is the number of harmonics, $\mathrm{V}$ is the core of the volume, $\mathrm{B}_{\mathrm{nr}}$ is Tangential component of air gap magnetic flux, $\mathrm{B}_{\mathrm{nt}}$ is Radial component of air gap magnetic flux.

In Formula 3 and 4, they show that the variation of the magnetic density has certain effect on the iron loss. For magnetic gear motor, the iron loss mainly occurs in the stator yoke of the outer rotor motor and the middle of the magnetic field. The magnetic field distribution of the motor is more complicated, the traditional method is not suitable for the iron loss calculation of the compound motor .

By the method of finite element analysis, the influence of the magnetic field in the end is neglected, and the iron loss of the composite motor with two different topologies is calculated. With the same rotation speed, the eddy current loss and hysteresis loss of the hybrid motor with two different topologies are shown in Figure 7.
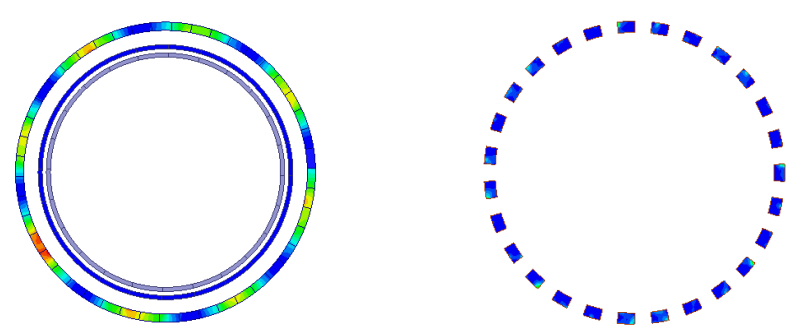

Fig. 7 loss distribution of the composite motor. 1 -Eddy current loss distribution, 2 -Loss distribution map of the magnetic field

The waveform of the air gap magnetic density and figure 7 show that composite motor yoke flux density of Halbach structure is lower than radial type rotor structure. In addition, although the magnetic flux density of general radial structure is larger, the harmonic volume of the air gap magnetic density is higher than that of the Halbach structure, Therefore, the application of Halbach structure of the composite motor has lower iron loss, higher transmission efficiency. The eddy current loss distribution of two kinds of composite motor is shown in Figure 8.

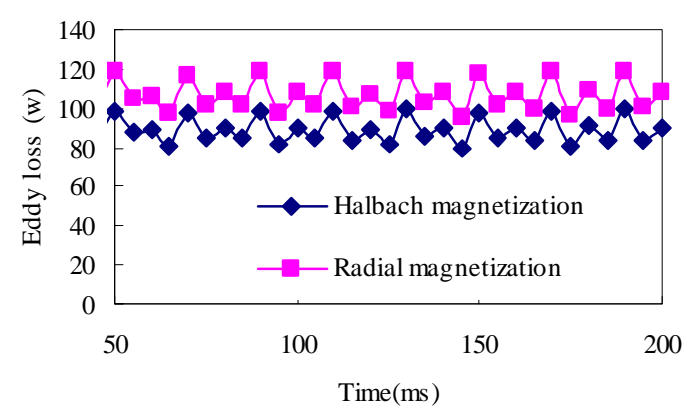

Fig. 8 Iron loss distribution of the composite motor

Figure 8 shows that eddy current loss is about $130 \mathrm{~W}$ when the permanent magnet radial magnetization. When using Halbach after magnetizing, the eddy current loss is about $100 \mathrm{~W}$, the eddy current loss is reduced about 30W. Due to the application of Halbach magnetization, harmonics of air gap flux density is reduced greatly, and the efficiency of the composite motor is improved. 


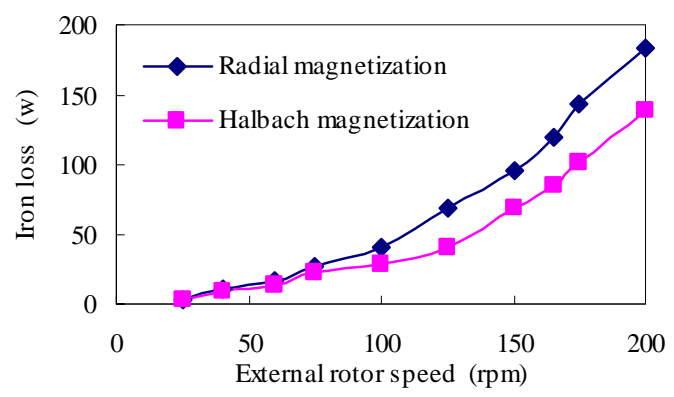

Fig. 9 No-load loss curve

Figure 9 shows the relationship between the no-load loss (iron loss) and the speed of the outer rotor, By the graph, the iron loss of the motor increases with the increase of the rotor speed of the composite motor, and by the application of Halbach magnetization method, the slope of iron loss curve is greatly reduced, and the load capacity of composite motor is increased.

\section{Optimization analysis of iron loss}

The amount of the iron loss will affect the operating efficiency of the magnetic gear motor, The eddy current loss will make the permanent magnet heating, produce irreversible demagnetization phenomenon, thereby affecting the performance of the motor. The influence of stator slot width on the permanent magnet eddy current loss is shown in Figure 10.

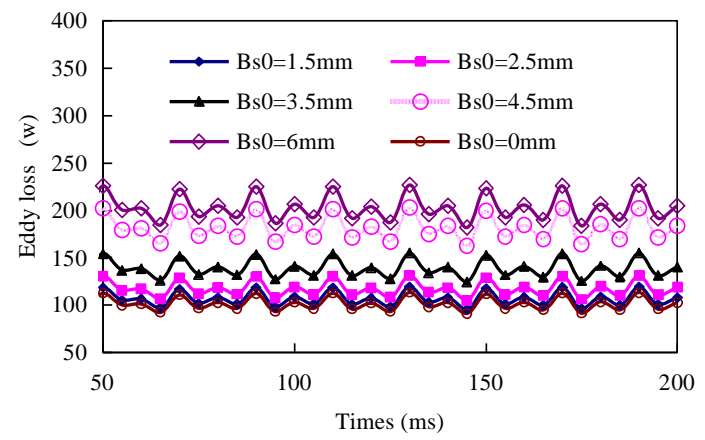

Fig. 10 Effect of slot width on eddy current loss

Figure 10 shows that with the decrease of the slot width, the eddy current loss of composite motor permanent magnet decreases gradually. But the slot width decrease will affect other motor operating performance as showing in table 1 . When the slot width decreasing, thecogging torque and eddy current loss of the motor are decreased, but the output of the motor is reduced. Comprehensively considering of the operation performance of motor, the best slot width is about $1.5 \mathrm{~mm}$.

Table 1 Effect of slot width on composite motor

\begin{tabular}{c|c|c|c}
\hline \hline Slot width $(\mathrm{mm})$ & Eddy current loss $(\mathrm{W})$ & Torque $\left(\mathrm{N}^{*} \mathrm{~m}\right)$ & Cogging torque $\left(\mathrm{N}^{*} \mathrm{~m}\right)$ \\
\hline 0 & 289 & 21.2 & 1.1 \\
\hline 1.5 & 344 & 24.9 & 1.5 \\
\hline 3 & 378 & 25.7 & 2.7 \\
\hline 4.5 & 443 & 26.3 & 4.8 \\
\hline 6 & 580 & 26.9 & 6.2 \\
\hline \hline
\end{tabular}

\section{Conclusion}

In this paper, proposed Halbach magnetization composite magnetic gear motor, transmission principle analysis of the motor, a motor finite element analysis model, and analysis of air gap magnetic field of the motor. Conclusion as below:

1. Halbach magnetic composite motor has higher coupling ability and transmission efficiency. 
2. The air gap magnetic sinusoidal of Halbach magnetized composite motor is higher harmonic is smaller, the amplitude of the flux density is relatively reduced. At the same time, the eddy current loss of is lower, the motor efficiency is higher. loading capacity is enhanced.

3. The stator slot width has an important effect on the eddy current loss, the smaller the notch, the smaller the eddy current loss, higher efficiency. But the size of the notch size affect the motor torque, the appropriate slot width need to be selected.

\section{Acknowledgements}

This work was financially supported by Anhui Education Department (gxbjzd2016098),Natural Science Fund of Anhui Education Department(KJ2014A282).

\section{References}

0[1] Du Shiqin, Zhang Yuejin, Jiang Jianzhong. Research on new type permanent magnet motor . Micro motor. 38(4).1-3(2010).

[2] Kyu Yun Hwang, Hai Lin, Se Hyun Rhyu. A study on the novel coefficient modeling for a skewed permanent magnet and overhang structure for optimal design of brushless DC motor.IEEE Transactions on Magnetics, 48(5).1918-1923(2012).

[3] Liu Xiping. Liu Shuai. Magnetic field finite element analysis of permanent magnet synchronous motor for elevator driver based on ANSYS . micro-machine, 44(4).16-18(2011).

[4] Zhang Dong, Zou Guotang, Jiang Jian medium. Design and research of a new type external rotor magnetic gear motor . Chinese Journal of Electrical Engineering, 28（30）,67-72(2008).

[5] Shen Jianxin, Wang Lili. Design and experiment of magnetic field modulated permanent magnet motor . Journal of electrical technology, 28 (11) ,28-34(2013).

[6] Jia Hu, Yingli Luo, Mingji Liu. Analysis of the Iron Loss of Line Start Permanent Magnet Synchronous Motor Using Time-Stepping Finite Element Method .IEEE,Trans.on magnetics. 40(6). 3237-3240(2010).

[7] Nakamura K, Fujimoto H, Fujitsuna M. Iron Loss and Magnetic Fields Analysis of Permanent Magnet Motors by Improved Finite Element Method with E\&S Model Electronics.Conference . IEEE, Trans.on magnetics. 37(5).3526-3529(2001).

[8] Kong Xiaoguang, Wang Fengxiang, Xu Yunlong. Analysis and calculation of iron loss of high speed permanent magnet motor . Motor and control journal,14(9).26-30(2010).

[9] Zhu Z Q. Evaluation of superposition technique for calculating cogging torque in permanent magnet brush less machines.IEEE,Trans.on magnetics. 42(5).1597-1603(2006).

[10] Nakamura K, Fujimoto H, Fujitsuna M. Torque ripple suppression control for PM motor with current control based on PTC.In.Proc Of Power Electronics.Conference (IPEC), Sapporo,1077-1082(2010). 